\title{
Mobile MSN Messenger: Still a Complement?
}

\author{
Marcus Nyberg and Didier Chincholle \\ Usability \& Interaction Lab, Ericsson Research, Stockholm, Sweden.
}

\begin{abstract}
In order to understand how mobile instant messaging services can fit into the users' current communication behavior, Ericsson Research performed a qualitative user study in Sweden in May 2007. The results showed that the respondents were positive towards (free of charge) mobile MSN Messenger and perceived it as an extension of the computer-based version that could be used anywhere. However, although MSN Messenger on the computer definitely was considered as a 'must-have' application, the mobile version was only perceived as a 'nice-to-have' application and a complement to text messaging (SMS). Almost one year later, in April 2008, Ericsson Research performed a short qualitative follow-up study with the same set of respondents to understand if and how the mobile MSN Messenger usage had changed. The results actually revealed that none of the respondents used mobile MSN Messenger anymore as the application no longer was free of charge. On a general level, the study highlights important considerations when introducing computer-based concepts and Internet services in a mobile environment.
\end{abstract}

Index Terms-User experience, mobile phones, IM, chat, text messaging, multi-device services

\section{INTRODUCTION}

On the Internet, instant messaging (IM) is a very popular communication method, not only among teenagers, giving users the ability to, e.g., exchange messages in real time. IM applications serve several purposes and are used to maintain social network status, to transfer files, and as an efficient communication tool $[5,6]$. In the mobile world, text messaging (SMS) is definitely among the most popular communication services in Europe and parts of Asia, allowing users to send a short one-way message to an individual or a group of individuals $[2,5]$. It is, however, assumed that Internet and multimedia services play an important role in the future mobile application development. Recently, we have also seen numerous services originally developed for computer-based Internet access being migrated to mobile phones. It is therefore believed that, e.g., IM applications will become an important and embedded part of future mobile communication [3, 10].

During the last years, the computer-based IM service providers have been expanding into the mobile domain and operators in several countries (e.g., France, South Africa, Sweden, UK, and US) offer IM services as part of their service packages. More advanced IM applications from, e.g., Microsoft, Yahoo, AOL, and Google have also been made available on mobile phones. In addition to these operator offers and the applications from more wellknown vendors, smaller independent companies have made mobile IM applications available for download and purchase to a large number of mobile phone models.
Of the many IM services available today, Ericsson Research chose to explore Microsoft's MSN Messenger in more detail. The major reason was that this IM service is one of the most popular in Sweden, but another important reason is that the service had been discussed in previous user studies (non-public information) that we wanted to compare the results with. In Sweden, the first embedded mobile MSN offer came from the operator 3 (mainly owned by Hutchinson Whampoa Ltd.) and the application was heavily advertised in the media during the first half of 2007. 3 were soon followed by the competing mobile operators Telia and Telenor, whom released their own MSN mobile applications during the summer of 2007.

In order to understand how mobile instant messaging services like mobile MSN can fit into the users' current mobile phone behavior [1, 9], Ericsson Research decided to perform a qualitative user study on mobile MSN from 3 starting in May 2007. This part of the work is referred to as the main user study throughout the paper. During this first part, several ideas for further work emerged; and shortly after the user study 3 did also make some changes in their offer that possibly could affect the mobile MSN usage. Therefore, Ericsson Research later decided to do a short qualitative follow-up study starting in April 2008. This time, the purpose was to learn more about how the changes that 3 had made actually had affected the respondents' perception of mobile MSN. The second part of the work is referred to as the follow-up study throughout this paper.

\section{MOBILE MSN FROM 3}

The recent versions of the MSN Messenger service are actually named Windows Messenger and Windows Live Messenger (the most recent name for the application), but we will use the old name here. The main reason is that the mobile service that we investigated was called MSN Messenger when it first was released and when we started this work. But another important reason is also that our respondents used (and still use) the name MSN Messenger, or simply MSN, when talking about the service.

At the time of the study, 3 offered free mobile MSN Messenger when purchasing a new phone or as part of other subscription and phone packages. The MSN application was pre-configured and accessible from the main menu when purchasing a new phone; and the Java client of the application was automatically downloaded when selecting mobile MSN for the first time. The application supported a subset of the functionality in the computerbased version, including the following basic functions:

- View and manage contacts with presence status.

- Point-to-point text chats.

- Change local presence status. 
An example of a more advanced feature that had been excluded is the highly rated file transfer [8], which is very central in the computer-based version of MSN Messenger. Multi-party chats, personal messages (free text), and personal images are other excluded features that are important in the computer-based version. At the time for the main user study in May 2007, 3 informed that these features were on the list of possible future improvements, but none of them were implemented when we conducted the study.

The application consists of two main tabs; the contact list (Fig. 1, left) and the conversation list (Fig. 1, right). In the two main tabs, the local user's presence status is always displayed at the top, e.g., 'Didier (Online). The contact list is sorted according to presence status and the conversation list is sorted by the most recent activity in the ongoing conversations (Fig. 1, left). Small speech bubbles at the top right hand side of the icon indicate ongoing conversations; and two lines symbolizing text appear in the bubble as soon as the user receives a new message.

A chat log (Fig. 2, left) opens up when a contact is selected either from the contact list or from the conversation list. In the chat log, the remote user's presence status is displayed at the top, e.g., 'Marcus (Online)'. Previous messages are listed in a conversational presentation style and new messages are highlighted with additional information about when the latest message is received. A small field for text input is also available at the bottom, allowing the user to enter text while viewing the chat log (Fig. 2, left).

Both the layout and the look and feel of the application are consistent with the computer-based version with one screen for the contact list, one screen for the conversation overview, and separated windows for each conversation (similar to the windows in the PC taskbar). From the More menu, the user can get additional information about contacts but also access the Settings menu (Fig. 2, right). The basic settings include possibilities to change, e.g., presence status, display name, and blocking properties.

Unlike the native messaging applications of the phone (e.g., SMS, MMS, or email), it is not necessary to keep the application displayed at the top of other applications. In fact, the Hide (minimize) option allows the user to keep mobile MSN running in the background while doing other tings on the mobile phone. A notification is automatically triggered as soon as a new message incomes; the user can easy retrieve the application with a key press if desired.

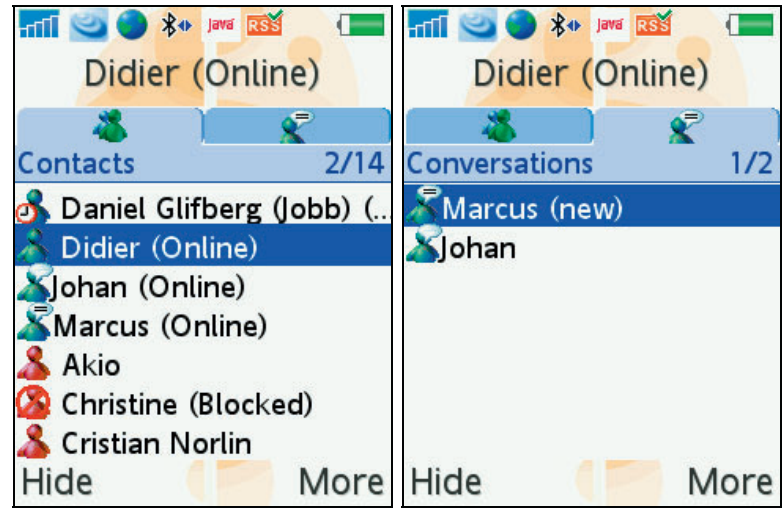

Figure 1. The two main tabs; the contact list (left) and the conversation list (right).

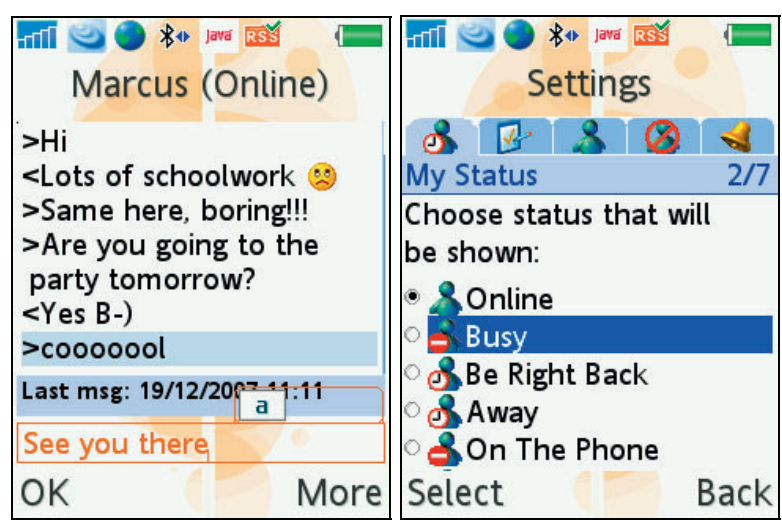

Figure 2. The chat log (left) and the settings tab for presence status (right).

\section{MAIN USER STUDY}

\section{A. Background and goals}

Prior qualitative research by Ericsson Research in Sweden [11] indicated that only a few respondents used IM services on the mobile phone although MSN on the computer was widely used. According to the respondents, MSN-like chatting on a mobile phone was initially perceived as too complicated and cumbersome to be used on such a tiny device. Furthermore, they were not willing to mix MSN contacts with mobile phone contacts.

These initial comments did not really fit to the way mobile MSN was presented in the advertisements and the apparent popularity of the application among certain user groups. With these aspects in mind, we outlined three main goals for the study:

- Find out why mobile MSN on 3's phones is becoming popular.

- Identify why, how, when, and where mobile MSN is used.

- Understand if mobile MSN is perceived as a substitute or a complement to SMS [4].

\section{B. Methodology}

The intention with the study was not to collect quantitative data; the approach was merely qualitative. The aim was to identify possible trends from a small and quite homogeneous group of Swedish mobile MSN users. The study was therefore carried out as semi-structured in-depth interviews on site in Stockholm, Sweden. Schools proved to be the appropriated place to run such interviews, as the respondents felt comfortable answering questions in a known (somewhat controlled) environment. This location also made it possible to briefly observe the respondents in their daily environment. The face-to-face sessions (Fig. 3) were all recorded on audio for later analysis.

Ten college and high school students (6 girls and 4 boys) in the age of 15-21 years participated in pairwise or single interviews. We chose this age group to be able to compare the findings with our previous work within the area; where we mainly used teenagers and young adults as respondents. The mobile MSN advertisements also indicated that this would be one of the main target groups for the service. The recruitment criterion was that the respondents must use mobile MSN from 3 regularly, which also implied that they used MSN on a computer. 


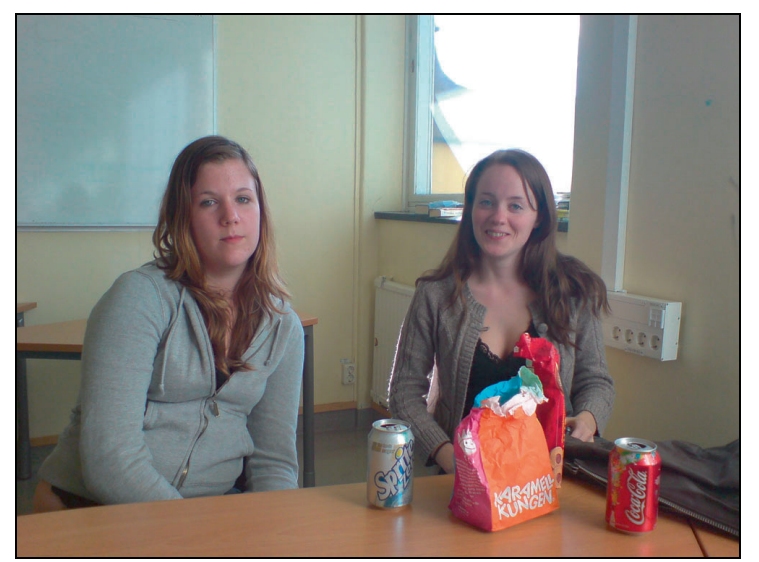

Figure 3. An example of the setting for a pairwise in-depth interview at a college school.

For the recruitment we simply visited a random selection of schools in the area around Stockholm city. No specific screening questions were used during the recruitment; we only made sure that the potential respondent in fact was a regular mobile MSN user with a 3 subscription. Although mobile MSN was advertised a lot in the spring of 2007, it is important to highlight that recruiting mobile MSN users at schools in Sweden proved to be more difficult than expected.

\section{Results}

\section{1) General}

First of all, it is important to stress that all respondents were heavy users of the computer-based version of MSN. From being used for social chit chatting with anyone at anytime, the application had also evolved into a more effective communication tool for a selection of friends. The respondents perceived MSN as a really important communication tool that was used for many different purposes, e.g., maintaining social networks, killing time, doing homework, and transferring files. Even though mobile MSN was only seen as a subset of the computer version, the respondents were positive towards it as an extension and a complement to the computer version - the application was available wherever they are [7].

The familiarity with the computer-based version of MSN Messenger was one contributing factor to why all of the respondents found mobile MSN quite easy to use. Another factor is that all respondents were very familiar with Internet, computers, mobile phones, and new technology. Many users in this age group have grown up with computers and Internet and can in many cases be characterized as digital natives [12].

During the interview, the respondents were asked to describe mobile MSN using the first three expressions that came to mind. The relative frequency of the most commonly mentioned expressions or words is displayed in Fig. 4. As it can be seen, the positive Fun was the most common expression followed by the positive Cheap, the positive Freedom, and the negative Drains the battery. The respondents' for mentioning these expressions will be further discussed in the sections below.

\section{2) Using mobile MSN}

Free of charge, or at least a very small flat rate charge, was expected for mobile MSN. And since this was the case for the application they used, it explains why Cheap was one of the more frequently mentioned expressions (Fig. 4). The complete application with all features is perceived as free on the computer; and it did not make much sense to the respondents to pay for a limited functionality in the mobile phone. It was clear that charging mobile MSN specifically may refrain several of the respondents from using it.

Mobile MSN was used at various locations when there was no computer at hand, e.g., at school, in the city, and at the bus/train. But also at home when the respondents were in a comfortable position and could not be bothered to go to the computer. These are the main reasons why Freedom was used to describe mobile MSN (see Fig. 4). The frequency of use varied from all the time (except for when logged in on a computer since it is not possible to be logged in from several devices at once) to a couple of times per week.

The respondents chatted with more or less the same persons as when using the computer-based version, i.e., close friends as well as distant friends that they would never send an SMS to. In fact, the informal conversation style made it easier to talk to anyone and the presence status triggered spontaneous communication. In general, the respondents did not change presence status as often as they do in the computer. It was common to log in to the application with the presence status 'Online', chat for a while, and then log out. Respondents that had mobile MSN running all the time used a presence status like 'Busy' or 'Appear Offline' to control possible incoming chats.

\section{3) Comparing Mobile MSN to SMS}

Mobile MSN had several advantages according to the respondents; instant messaging was perceived as a more direct and quicker way of communicating - it was like having a (real-time) conversation. The presence status clearly indicated who is available for chat and if an answer could be expected, which made the application very suitable for killing time situations. In Fig. 5, one of the comments from a respondent called Lisa shows how easy it can be to just pick up mobile MSN and start a communication with someone that was logged in.

It was socially accepted to start a chat with anyone that is available, unlike for SMS where the receiver is often selected before starting the application. The unlimited number of characters provides more freedom for selfexpression, especially when texting with distant/new friends. All these examples illustrates why the respondents perceived mobile MSN as Fun to use (see Fig. 4).

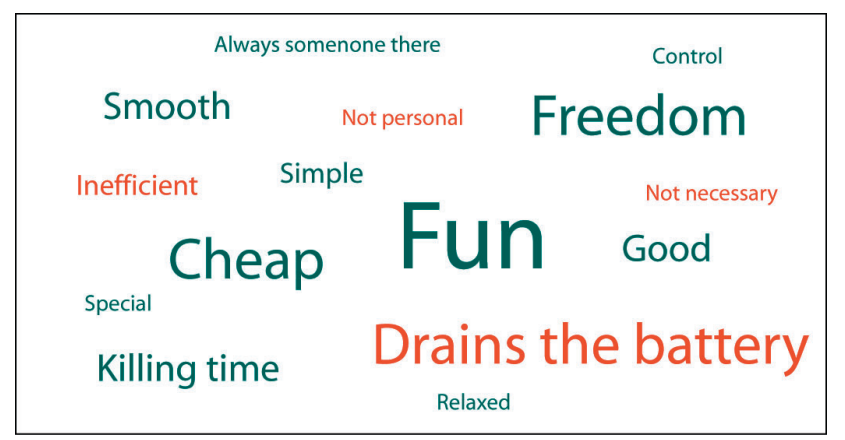

Figure 4. The relative frequency of common words to describe the service, larger text means more frequently mentioned words. Green indicates positive words and red negative words. 


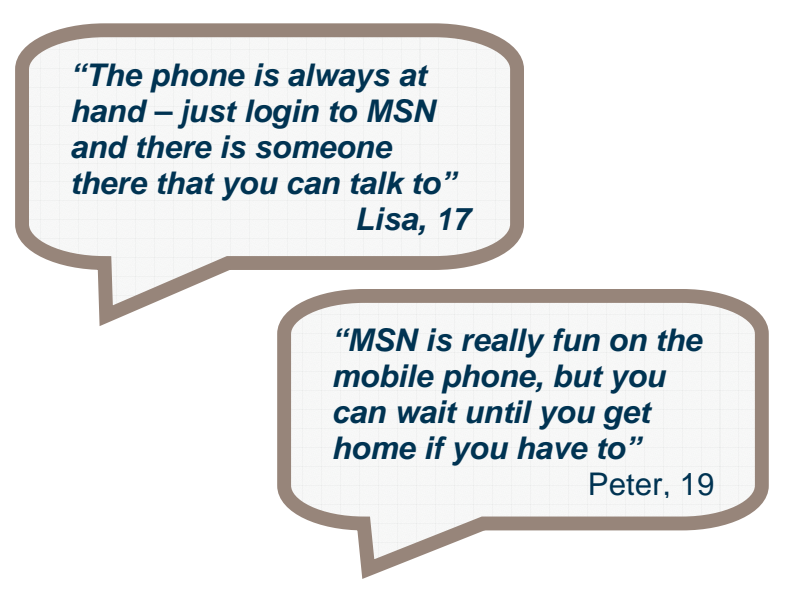

Figure 5. Some significant comments from two of the respondents describing their positive experiences of mobile MSN.

Being able to reach friends on a computer was another advantage; the number of potential friends to communicate with increased for the respondents. The critical mass for an Internet service like MSN was not an issue as the contact list was the same as on the computer and therefore already populated (one account for multiple devices). MSN Messenger was seen as one application that could be accessed from either the computer or from the mobile phone.

Although the respondents were satisfied with mobile MSN and saw several advantages with using it, SMS was still frequently used. The respondents were actually sending lots of SMSs, either for a more defined and important purpose or occasionally back and forth in a chat manner. Using SMS was also perceived as a more personal way of communicating and mainly for close friends. One reason was that the mobile phone number was much more private than an MSN address (email address) and therefore had a greater value; not all of the respondent's MSN contacts shared their phone number with the others. You also need to know each other to better understand the meaning of the SMS, which is limited in length and therefore less suitable for distant friends.

The communication with distant friends that the respondents only could (or wanted to) reach through MSN was seldom time critical - it could always wait until being at home. In Fig. 5, one of the comments from a respondent called Peter clearly demonstrates this point. On the other hand, the communication with close friends could not always wait and sometimes required using SMS. According to the respondents, one major advantage with SMS was that users could send a one-way message, feel confident that the message reaches the receiver, and move the attention from the mobile phone to another activity. With SMS it was not the same pressure to answer right away and the conversation was clearly based on turn-taking, making the communication easier to control in a mobile environment. An SMS did not necessarily require an immediate answer from the receiver.

\section{4) The Mobile Environment}

For mobile MSN, the respondents had to consider limiting factors that did not exist when using MSN on a computer or when using SMS, e.g., the battery consumption and the network coverage. A drained battery made all communication impossible; therefore a specific amount of battery always had to be allocated for major communica- tion services like SMS and voice calls. This is why 'Drains the battery' was the most frequently mentioned negative expression by the respondents (Fig 4). Poor network coverage was another problem that could result in interrupted and lost conversations, and eventually lead to irritation and frustration.

The mobile environment was also full of distractions that easily could cause a user to loose focus. The respondents often focused on a single conversation instead of having parallel conversations as on the computer. Due to the limitations of their mobile device, multiple application windows were not possible and it was quite cumbersome to have several conversations running in the background. Minor problems were caused by the fast interaction pace of MSN, which could be stressful even in a computer environment. To keep up in a mobile environment, the respondents had to dedicate more focus and attention on the actual input [7]. Messages from mobile devices were naturally a bit shorter, making the conversations a bit limited and not as rich as on a computer.

\section{5) Suggestions for improvements}

The respondents were asked to come up with a few suggestions for improvements. Unsurprisingly, they compared mobile MSN to the computer-based version and wanted to have more advanced functions in the phone as well. On the top of their list were mainly a number of features that had been removed from the mobile version:

- Feedback when a remote contact is typing a message; an indication that the remote mobile user is typing a message can make the communication less stressful.

- Possibility to log in with a presence status of your choice; it is considered as very important for privacy reasons and to be able to be in control of the communication

- Possibility to save a conversation log; it is considered as very important to remember what has been decided after having closed the mobile MSN application.

- Possibility to access the mobile chat history from the computer-based application; this would make the mobile and computer experience more integrated and seamless.

- Images and music sharing; as file transfer widely used in the computer version, this could provide an additional value when in a mobile environment.

- Less frequently suggested improvements were:

- Allowing multi-party chat; even though this is seldom used on the computer it can provide very efficient group communication from a mobile device

- Indicating the type of device that contacts are using; this information is included in the computer-based version and works like an extended presence to know more about the remote users' situation and capabilities.

- Displaying personal images and free text into the contact list; such fun features make it easier to express moods and activities.

\section{FOLLOW-Up USER STUDY}

\section{A. Background and goals}

In the beginning of September 2007, 3 in Sweden started to charge the customers 19 SEK (approximately 2 
Euro) per month for the mobile MSN service; a cost that was added on the top of post-paid subscriptions. About the same time, the other operators in Sweden offering mobile MSN did also start charging the customers a similar amount. The mobile MSN application from 3 was not updated in any major way when the charging was introduced. As previously mentioned, the main user study in 2007 reveled that the majority of the respondents were very positive, but also that the price was an extremely important factor. Starting from these findings, we outlined three main goals for the follow-up study in April 2008:

- Find out if the respondents still use mobile MSN on 3's phones regularly,

- Identify if the usage and user perception of the mobile MSN application have changed over the year. Was the application perceived as a complement to SMS now or had it only been a fad for a short period of time?

- Understand if and how the charging (19 SEK per month) for the service has affected the usage of mobile MSN.

\section{B. Methodology}

The setup of follow-up study was just like the main user study an entirely qualitative one. Since we had already established a contact with the respondents, we used a very simple approach for this part. We contacted the respondents from the previous year either using email or Facebook. Once we had gotten hold of the respondents, email conversations were used to send a set of specific questions. If there were anything unclear in the respondents' answers, a second email asking for clarifications was sent. The same applied if the answers revealed anything unexpected that we wanted to know more about, which means that the email interviews were done in a somewhat semistructured manner. Seven out of the ten original college and high school students (3 girls and 4 boys) in the age of 17-22 years answered the email questions completely; one respondent only answered the most important question about mobile MSN usage.

\section{Results}

\section{1) Mobile MSN Usage}

All of the eight respondents in the follow-up study had stopped using mobile MSN. The main reason for stopping to use the services was that 3 started to charge for mobile MSN and that the respondents did not want to, or could not be bothered, to pay for the service. Some respondents had lost their phones or changed their subscription and when they got their new phone or subscription, mobile MSN was not included. The great majority of respondents mentioned that they would consider starting to use mobile MSN again if it was included in the subscription for free. In Fig. 6, one of the comments from a respondent called Jonas shows that he experienced that it was not even possible not use MSN anymore - paying for it was not an option he had considered at all.

MSN Messenger on the computer was still widely used by the respondents and they all still saw it as an important computer application for communicating. However, the majority estimated that they use it to a slightly lesser extent than one year ago. A few of the respondents mentioned that social network services like Facebook, My Space, and You Tube has taken over some of the MSN Messenger usage.

\section{2) Mobile MSN Perception}

The respondents' opinions regarding the perceived difference between mobile MSN and SMS had not changed. Mobile MSN is an application mainly used for fun and for killing time. SMS, on the other hand, is a must-have application used for communication when they have something specific to say or to talk about. Some respondents started to use SMS a bit more after they stopped using mobile MSN, but this does not necessarily depend on mobile MSN. Several respondents had a flat rate subscription with unlimited SMSs and in Fig. 6, one of the comments from a respondent called Jenny explains how appreciated this feature is.

Very few of the respondents' friends used mobile MSN and the general impression was that mobile MSN was not as hip as a year ago. Some of the respondents mentioned that lots of things can happen during a year when you are a teenager or young adult, both in terms of Internet services and in the personal life. New creative Internet and mobile services pop up all the time; and during the last year the respondents thought that Facebook has made one of the most significant entrances on the market. Several respondents explained how they had moved between social networks when growing up; different communities and applications are popular in different age groups. Some respondents had also started working extra, which means that there was much less time to kill. All these are examples that may have affected the mobile MSN popularity in Sweden.

Furthermore, some of the respondents had started to use mobile Internet services, e.g., the public transportation time tables, and a few respondents had also tried social network services like Facebook mobile. However, none of them had replaced the mobile MSN usage and although being exposed to several new services during the year the respondents' suggestions for improvements had not changed significantly. File transfer was still on the top of the list, and the possibility to see images of persons and to have multi-party chats were frequently mentioned.

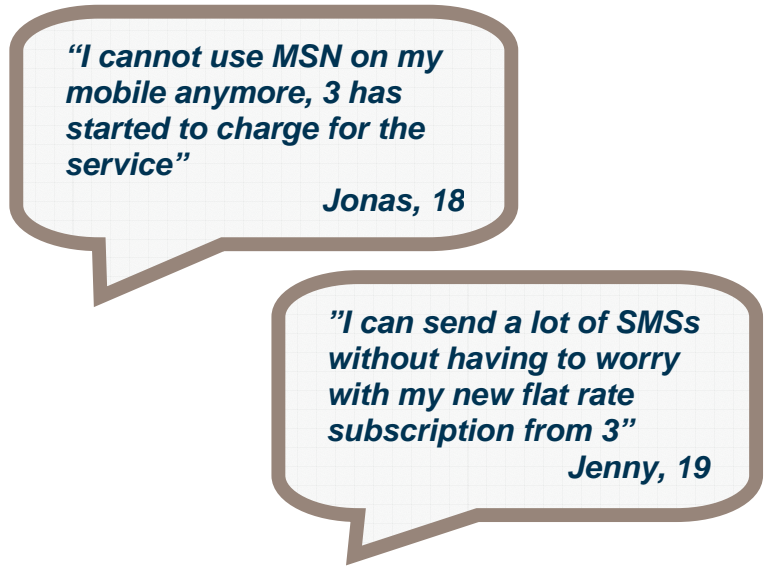

Figure 6. Some significant comments from two of the respondents describing their experiences of being charged for mobile MSN.

\section{DISCUSSION}

Although (free) mobile MSN was popular with our respondents, there was no real peer pressure from the group to acquire and use the application on the mobile phone. The respondents did not expect their friends to be always available on mobile MSN; and using mobile MSN was not 
required to maintain a good image among peers [15]. They respondents used, e.g., SMS, for the daily communication with close friends and the communication with more distant friends could wait until being at home. Communication between mobile MSN and MSN Messenger on a computer was experienced as a bit uneven and not optimal; it would be beneficial to communicate with someone also using mobile MSN.

It is important to point out that this study was conducted only in Sweden and that the situation can be different in other countries. For instance, in some Asian countries where stationary Internet access is less common and a mobile phone is the first Internet experience for some users [13]. In these cases, mobile applications and services do not suffer from the same level of comparison with computer-based applications. There are also countries like Japan where Internet services for various reasons have gained usage in mobile environments [14]. An evolution like this can potentially mean that a service like mobile MSN eventually becomes an own communication tool, not only used to killing time.

Lately, more advanced mobile phones with full QWERTY keyboards have also become very popular. This type of mobile phones that resembles small computers can be much more efficient for instant messaging, making the use anytime and anywhere more suitable from an interaction and usability point of view. This aspect may also affect the future view of IM applications like mobile MSN.

Something that may have affected the interest for mobile MSN is that the mobile operators in Sweden only advertised mobile MSN for a limited period of time, then shifting to other possible killer applications. Mobile TV, videos, music, email, maps, and audio books are some examples of services that have been advertised during the last year instead of mobile MSN. This means that mobile MSN had a quite short exposure in the Swedish mass media. Between the time we performed the first and the second set of interviews (i.e., one year), various computerbased Internet services like Facebook and Twitter also attracted a lot of attention. This may have affected the view of MSN Messenger in general, making the application less cool to use. Previous Ericsson Research studies (non-public information) have also indicated that users tend to move from service to service; similar to the way certain clubs and bars are gaining or loosing popularity within different groups of people.

The respondents in the study were clearly not willing to pay 19 SEK (approximately 2 Euro) per month for the current version of the mobile MSN application. They did not believe that the additional value of having the service in the mobile phone as well was sufficient. The question is how this additional value can be created. Today, the mobile version of MSN Messenger only provides a limited version of the computer-based version that one needs to pay for on top of the regular subscription. For the current mobile MSN application, the urgency and convenience does apparently not outweigh the uncertainty of usage and the expectation that it should be free. The latest release of 3's mobile MSN came out in May 2008 (unfortunately it was not ready to use in time for the study). This updated version encloses many of the improvements that the respondents have mentioned, e.g., send and receive smaller files, view personal messages (free texts), and have multiparty chats. According to this study, these features are obviously required for mobile MSN to become a musthave application. But even with these changes mobile MSN is still a limited version of the computer-based version, which is available for free. The main issue remains to understand if the changes are enough to make the respondents willing to pay, e.g., 19 SEK per month.

On a general level, the results from the study highlight several important aspects when migrating Internet services to mobile phones. Besides considering the traditional usability and utility values, the designers must do more than simply shrink the service. They must mobilize the user experience by adapting the application to the mobile user's situation and environment, and ask themselves how the mobile environment affects the perception of the different functions, i.e., how can the mobile version of the application provide an additional value? When looking at mobile communication services, the characteristics of the users' social network and current communication behavior must also be carefully considered. The users have likely adapted certain strategies for different types of mobile communication methods to suit different purposes. Any new service should be carefully integrated into this behavior and these strategies in order to become a key mobile communication service.

Finding ways to provide an additional value compared to the computer-based version and not just being a simplified version could be one way for mobile MSN-like applications to become a complement, or even more than a complement. It would be therefore interesting to explore how mobile values like location and proximity can override expectations for free-of-charge services.

\section{CONCLUSIONS}

At the time for the main user study (May 2007), mobile MSN was not perceived as a main communication option for the respondents (10 Swedish users in the age of 1521). SMS and voice calls were still more important communication applications in the mobile phone. While MSN on the computer definitely was a 'must-have application', mobile MSN was only a 'nice-to-have application' that could be used for fun as a complement to the more valuable SMS service. The follow-up study (April 2008) showed that mobile MSN was not a communication option at all for the respondents anymore. The fact that the operator had started charging for the service had caused them all to stop using it.

The main user study clearly indicated that a welldesigned mobile IM service including presence status and a conversational presentation, has a value for many users. It can make the mobile communication more alive, straight-forward, and exciting to use. But the follow-up study showed that the respondents kept their mental models from the stationary Internet to the mobile phone; an Internet application that is free from a computer should be free on the mobile phone as well.

Finally, an attempt to answer the question in the title: Is mobile MSN still a complement? According to our Swedish respondents, the previous version from 3 was not a complement at the time of the study. The latest version from 3 may become a complement as it includes many of the suggested improvements. But for mobile MSN to become more than a complement, we believe that the service must evolve into something different from the computerbased version. 


\section{ACKNOWLEDGMENT}

The authors would like to thank Caroline Hägglund at Usability \& Interaction Lab, Ericsson Research, for valuable help with summarizing the respondents' answers from the follow-up interviews and giving input to the analysis phase.

\section{REFERENCES}

[1] M.E. Björling, J. Carlsten, P. Kessler, E. Kruse, and M. Stille, “Sharing everyday experiences”, Ericsson Review, No. 1, 2006.

[2] M. Björn, "The Right of Interpretation”, in Information Communication Technologies and Emerging Business Strategies, S. Van Der Graaf, Y. Washida, Eds, New York, Idea Group Inc, 2006, pp 36-58.

[3] S. Ellison, "Mobile Instant Messaging: The Next Major Mobile Opportunity”, White Paper, IDC, Framingham, 2007.

[4] R. E. Grinter, L. Palen, and M. Eldridge, "Chatting with Teenagers: Considering the Place of Chat Technologies in Teen Life”, in ACM Transactions on Computer-Human Interaction, Vol. 13, No. 4, 2006, pp 423-447.

[5] A. Lenhart, M. Madden, and P. Hitlin, "Teens and Technology, Youth are leading the transition to a fully wired and mobile nation", in Pew Internet and American Life, 2005 (available from http://www.pewinternet.org/pdfs/PIP_Teens_Tech_July2005web.p df).

[6] C. Lewis and B. Fabos, "Instant Messaging, literacies, and social identities”, in Reading Research Quarterly, Vol. 40, No. 4, 2005, pp 470-501.

[7] S. Nylander, "Real-Life Use of Multi-Device Services”, Technical report 2006:18, Swedish Institute of Computer Science, Stockholm, 2006.

[8] E. Shiu, E. and A. Lenhart, "How Americans use instant messaging”, in Pew Internet and American Life, 2004 (available from http://www.pewinternet.org/pdfs/PIP_Instantmessage_

Report.pdf).
[9] K. P. Tang and J. I. Hong, "Using current SMS and mobile IM practices to inform social mobile application design”, Presented at CHI'2006, workshop on Mobile Social Software, 2006.

[10] H. Verkasalo, "Empirical Observations on the Emergence of Mobile Multimedia Services and Applications in the U.S. and Europe”, in Proceedings of MUM'06, ACM, Stanford, 2006.

[11] D. Chincholle, M. Björn, C. Norlin, and M. Lindqvist, "Chat on a phone, not a PC clone: IMS-based mobile community service”, Ericsson Review, No. 1, 2008 (available from http://www.ericsson.com/ericsson/corpinfo/publications/review/20 08 01/files/2 Chat on phone.pdf).

[12] M. Prensky, "Digital Natives, Digital Immigrants", On the Horizon, MCB University Press, Vol. 9 No. 5, 2001.

[13] D. Joshi and V. Avasthi, "Position Paper - Mobile Internet UX for Developing Countries”, in Proceedings of Mobile HCI 07, Singapore, 2007.

[14] M. Ito, "Personal, Portable, Pedestrian", Mobile Phones in Japanese Life, M. Ito, D. Okabe, and M. Matsuda, Eds, Cambridge, MIT Press, 2005.

[15] S. McClatchey, "The Consumption of Mobile Services by Australian University Students”, in International Journal of Mobile Marketing, Vol. 1, No. 1, June 2006

\section{AUTHORS}

Marcus Nyberg (marcus.nyberg@ericsson.com) is working as a research engineer within user experience at Usability \& Interaction Lab, Ericsson Research, 16480 Stockholm, Sweden.

Didier Chincholle (didier.chincholle@ericsson.com) is working as a senior specialist in interaction design for mobile services at Usability \& Interaction Lab, Ericsson Research, 16480 Stockholm, Sweden.

This article was modified from a presentation at the 21st Symposium on Human Factors in Telecommunication (HFT 2008) in Kuala Lumpur, Malaysia, March 2008. Manuscript received 2 September 2008. Published as submitted by the authors. 